\title{
Promoção da Saúde e Nutriçáo Infantil na Atenção Básica
}

\section{Health promotion and pediatrics nutrition in primary care}

\author{
Alessandra Amorim Machado ${ }^{1}$ \\ Fabio Aguiar Alves ${ }^{2}$
}

\section{Resumo}

Objetivo: Promover uma revisão da literatura sobre a promoção da saúde na infância e adolescência e apresentar estratégias para prevenção da obesidade infantil a nível de atenção primária.

Fontes dos dados: Revisão nas bases de dados MEDLINE, SciELO e LILACS no período de 2000 a 2012. Os seguintes critérios de inclusão foram levados em consideração: trabalhos publicados entre 2000 e 2010, que abordassem nutrição infantil, obesidade infantil e estratégias para promoção da saúde nesta faixa etária. Além disso, foram selecionados livros que abordassem o tema da atenção básica em pediatria.

Síntese dos dados: Os erros alimentares e o sedentarismo caracterizam a fase atual da história da humanidade aumentando o número de doenças crônicas não-transmissíveis. Para promoção da saúde é necessário mudança de comportamento da população de forma a assumir a responsabilidade individual pela prática de atividade física regular e alimentação adequada.

Conclusão: A nutrição infantil emerge como prioridade no horizonte da saúde pública e o pediatra como instrumento fundamental na promoção da saúde e prevenção de doenças.

Pavavras-chave: Promoção de saúde, nutrição infantil, obesidade.

\section{Abstract}

Objective: To promove a review of the literature on health promotion in childhood and adolescence and present strategies to prevent childhood obesity in primary care.

Sources: Review of MEDLINE, SciELO and LILACS databases in the period 2000 to 2012. The inclusion criteria were considered: articles published between 2000 and 2010 that focused on child nutrition, childhood obesity and strategies for health promotion in this age group. Books about healthy primary care in pediatrics were also selected.

Summary of the findings: Uncorrected diet practices and sedentary lifestyle represents the current moment of humanity history, increasing the numbers of non transmitted chronic diseases. For health promotion is necessary a change in people behavior accepting own responsibilities for regular exercise practice and correct diet.

Conclusion: Children nutrition appears as a priority in public health and the pediatrician has a fundamental participation on health promotion and disease prevention.

Keywords: healthy promotion, children nutrition, obesity.

\footnotetext{
${ }^{1}$ Mestranda. Mestrado Profissional em Ensino em Ciências da Saúde e do Meio Ambiente -UniFOA

${ }^{2}$ Professor Doutor, Programa de Patologia - Universidade Federal Fluminense-UFF
} 


\section{INTRODUÇÃO:}

A Atenção Primária à Saúde (atenção básica), assim expressa na Declaração de Alma-Ata, já era apontada como a "chave" para o cumprimento de uma das principais metas dos países, das organizaçóes internacionais e de toda a comunidade mundial para o ano 2000 - o alcance, por parte de todos os povos do mundo, de um nível de saúde que lhes permitisse levar uma vida social e economicamente produtiva (OMS, 1978, apud JAIME et al, 2011).

Os cuidados primários com a saúde, conforme Declaração de Alma-Ata são cuidados essenciais de saúde baseados em métodos e tecnologias práticas, cientificamente fundamentadas e socialmente aceitáveis, ao alcance universal de indivíduos e famílias, da comunidade, mediante sua plena participação e a um custo que a comunidade e o país possam manter. Entre os cuidados primários estão a promoção da nutrição apropriada e os cuidados de saúde materno-infantil.

Segundo Jaime et al (2011), os grandes marcos da Atenção Básica no Brasil foram a criação do Programa de Agentes Comunitários de Saúde em 1991 e o Programa de Saúde da Família, agora nomeado Estratégia de Saúde da Família, em 1994; os quais se constituíram nas principais estratégias para organização das açóes da Política Nacional de Atenção Básica em 2006.

A Atenção Básica é caracterizada por um conjunto de açóes de saúde que abrangem a promoção e a proteção da saúde, a prevenção de agravos, o diagnóstico e o tratamento. É desenvolvida com o trabalho em equipe e dirigida a populaçôes localmente definidas, visando a resolver os problemas de saúde de maior freqüência e relevância em seu território (BRASIL, 2006). É o contato inicial dos usuários com os sistemas de saúde e considera o sujeito em sua singularidade, complexidade, integralidade e inserção sociocultural.

A Política Nacional de Alimentação e Nutrição (BRASIL, 2003) e a Política Nacional de Promoção da Saúde (BRASIL, 2006) prevêem açóes específicas para a promoção da alimentação saudável. Em 2011, foi lançado pelo Ministério da Saúde o Plano de Ações Estratégicas para o Enfrentamento das Doenças Crônicas Não Transmissíveis (2011-2022), entre as quais está a obesidade (BRASIL, 2011). Neste Plano estão previstas as açóes de vigilância, informação, avaliação e monitoramento, promoção da saúde e do cuidado integral, sendo a Atenção Básica o espaço preferencial para o desenvolvimento de açôes voltadas à promoção da saúde e à prevenção da obesidade, aumentando-se a necessidade de formaçáo de profissionais aptos a colocar em prática as açóes propostas.

Entre os objetivos da promoção de saúde está o de enfatizar a mudança de comportamento da população de forma a internalizar a responsabilidade individual da prá- tica de atividade física regular e a promoção à alimentação adequada e saudável.

Atualmente, vivemos uma situação conhecida como transição nutricional, caracterizada pela inversão da distribuição dos problemas nutricionais da população, com uma passagem da desnutrição para a obesidade. As mudanças que vêm ocorrendo nos padróes do consumo alimentar, como os hábitos inadequados, principalmente na infância e na adolescência, têm levado pesquisadores e profissionais a indicar a necessidade de intervençóes nesse quadro (FAGLIOLI; NASSER, 2008, apud SILVA \& BITTAR, 2012). Diante deste contexto, este artigo teve como objetivo fazer uma revisão da literatura sobre a promoção da saúde na infância e adolescência e apresentar estratégias para prevenção da obesidade infantil a nível de atenção primária em saúde.

\section{MÉTODOS E CRITÉRIOS DE BUSCA DE ARTIGOS}

Para a seleção dos artigos analisados no preparo desta revisão, recorreu-se à pesquisa sistemática das bases de dados MEDLINE, SciELO e LILACS no período de 2000 a 2011. Os termos utilizados na busca foram: promoção de saúde, nutrição infantil e obesidade. Os seguintes critérios de inclusão foram levados em consideração: trabalhos publicados entre 2000 e 2010 que abordassem nutrição infantil, obesidade infantil e estratégias para promoção da saúde nesta faixa etária. Além disso, foram selecionados livros que abordassem o tema da atenção básica e obesidade em pediatria. Foram incluídos os estudos nas modalidades coorte prospectivo ou retrospectivo, artigos de revisão sistemática e não sistemática e somente artigos escritos em língua portuguesa e inglesa. Como critérios de exclusão: estudos envolvendo populaçáo adulta, editoriais de revistas, relatos de casos isolados, artigos recuperados pelos termos de busca que não tinham relação com o assunto da revisão e em língua diferente das citadas para inclusão.

\section{RESULTADOS E DISCUSSÃO}

\section{A obesidade infantil}

A obesidade é uma doença complexa que apresenta graves dimensóes sociais e psicológicas, afeta praticamente todas as faixas etárias, grupos socioeconômicos e é considerada uma doença não transmissível (LOPES, PRADO \& COLOMBO, 2010). Na infância corre mais freqüentemente no primeiro ano de vida, entre 5 e 6 anos e na adolescência (DIETZ, 2001; EBBELING et. al, 2002; citados por MELLO, 2004). 
Através da revisão de literatura foi possível verificar que a obesidade tem sido considerada atualmente um dos principais problemas de Saúde Pública. O aumento epidêmico na prevalência de obesidade em diferentes populaçóes e faixas etárias tem despertado cada vez mais o interesse em esclarecer suas causas e conseqüências (FILGUEIRAS et al,2012).

Segundo Miranda e Navarro (2008), trata-se de doença multifatorial, crônica e cujo tratamento é um desafio, sendo este o motivo pelo qual as crianças constituem o principal grupo para prevenção. Para Rodrigues e Boog (2006), diversos trabalhos são publicados sobre obesidade, mas quase não há estudos que busquem o aprimoramento das estratégias educativas para prevenção. E prevenir a obesidade significa diminuir, de forma racional e menos onerosa, a incidência de doenças crônico-degenerativas (SAHOTA et. al, 2001; SBP, 2008; PEREIRA, 2006).

Segundo Monteiro e Conde (2007), atualmente transbordam os excessos a impedirem a vida humana saudável. Estima-se que, no Brasil, haja cerca de três milhôes de crianças, com idade inferior a 10 anos de idade, com excesso de peso. Desses, $95 \%$ estariam relacionados à má alimentação, enquanto apenas 5\% seriam decorrentes de fatores endógenos. Segundo Corso et al (2003), apesar do fato da obesidade ser ainda prevalente em crianças da classe média e alta, é crescente seu surgimento entre crianças pobres. Este aumento da prevalência de obesidade em crianças de diferentes faixas econômicas está relacionado a mudanças no estilo de vida (outros tipos de brincadeiras, mais tempo frente à televisão e jogos de computadores, maior dificuldade de brincar na rua pela falta de segurança) e nos hábitos alimentares (maior apelo comercial pelos produtos ricos em calorias, maior facilidade de fazer preparaçóes industrializadas e menor custo de produtos de padaria) (ABRANTES, 2002; WANG et. al, 2002, MIRANDA \& NAARRO, 2008; FIATES, AMBONI E TEIXEIRA, 2008).

As complicaçôes metabólicas decorrentes da obesidade interferem diretamente na qualidade de vida, representam maior risco para o futuro desta população e, se não trabalhadas adequadamente, podem desencadear repercussóes psicossociais.

É consenso que a obesidade infantil vem aumentando de forma significativa e que determina complicaçôes na infância e na idade adulta. Segundo Mello et al (2004), na infância o manejo pode ser ainda mais difícil do que na fase adulta, pois está relacionado a mudanças de hábitos e disponibilidade dos pais, além de uma falta de entendimento da criança quanto aos danos da obesidade. No entanto, as mudanças devem ser estimuladas, pois a infância é a faixa etária mais importante para a aprendizagem dos princípios que norteiam a nutrição adequada (CASTRO et. al, 2002).
Crianças têm poucos conhecimentos em nutrição e hábitos alimentares saudáveis, sendo importante a educação nutricional como estratégia de combate à obesidade, com ações integradas envolvendo famílias, escolas, comunidade e a indústria de alimentos (OLIVEIRA E FISBERG, 2003; TRICHES \& GIULIANI, 2005).

Promover uma alimentação saudável implica desenvolver mecanismos que apóiem os sujeitos a adotar modos de vida saudáveis, revendo hábitos alimentares, num contexto em que a globalização e a urbanização caracterizam-se como movimentos dificultadores (COUTINHO, GENTIL \& TORAL, 2008). Assim, torna-se fundamental associar medidas de incentivo como estratégias de informação, campanhas e educação alimentar e nutrição; com medidas de apoio que auxiliem os indivíduos a fazerem suas escolhas mais saudáveis (rotulagem nutricional dos alimentos, incentivos voltados à produção dos alimentos, comercialização, abastecimento e o acesso físico a alimentos mais saudáveis); com medidas de proteção que implicam medidas regulatórias que protegem a população de práticas abusivas, como as de marketing e publicidade de alimentos calóricos, focadas ao público infantil (UAUY \& MONTEIRO, 2004; BRASIL, 2006).

\section{O PAPEL DO PEDIATRA NA ATENÇÃO BÁSICA}

Segundo a Sociedade Brasileira de Pediatria (2008), a promoção, proteção e recuperação da saúde do ser humano durante a fase de crescimento e desenvolvimento definem a pediatria como a especialidade médica que abrange um conteúdo científico singular. É atribuição comum a todo profissional da área realizar o cuidado em saúde desta população, garantir a integralidade da atenção por meio da realização de ações de promoção da saúde, prevenção de agravos e medidas curativas.

A prevenção primordial visa prevenir que as crianças se "tornem de risco" para sobrepeso; a prevenção primária objetiva evitar que as crianças "de risco" adquiram sobrepeso; e a prevenção secundária visa impedir a gravidade da obesidade e reduzir as co-morbidades de crianças com sobrepeso e obesas. As iniciativas de prevenção primordial e primária são as mais eficazes, principalmente se forem iniciadas antes da idade escolar e mantidas durante a infância e a adolescência (YANOVSKI, 2002).

A avaliação do estado nutricional é uma estratégia fundamental para prevenção do ganho de peso durante a infância e realizar o acompanhamento das alteraçóes nutricionais é fator primordial para a promoção da saúde. Segundo REICHERT et al (2012), no programa de vigilância em saúde da criança, a atuação do profissional deve ser integral, 
e o foco da atenção é o de não perder oportunidades de atuação, seja na prevenção, como na promoção e/ou assistência, mantendo o vínculo com a família e estimulando a responsabilidade contínua e conjunta (serviço e família) no cuidado à criança.

Vários programas fazem parte do modelo de vigilância à saúde da criança, como o de imunização, a detecção de abusos e violência contra a criança, a promoção da saúde e de hábitos de vida saudáveis, o monitoramento de algumas doenças crônicas, além da vigilância do crescimento e desenvolvimento. Nesta perspectiva, cada contato entre esta e o serviço de saúde, independente do motivo, deve ser tratado como uma oportunidade para análise integrada e preditiva de sua saúde e para uma ação de promoção da saúde com caráter educativo (FIGUEIRAS et al, 2003), pois muitas crianças que procuram os serviços de atenção básica em saúde se encontram em risco nutricional e vivem em condiçôes ambientais adversas.

Segundo Escrivão e Taddei (2007) quanto mais precoce a aquisição de hábitos alimentares corretos, mais sadia a população de adolescentes e adultos de uma sociedade. Daí a importância da participação ativa do pediatra identificando fatores de risco familiares, avaliando e monitorando através da puericultura, esclarecendo aos pais sobre a educação alimentar de seu filho, verificando desvios na dinâmica familiar que influenciem o comportamento alimentar da criança, estimulando a adesão dos pais a um estilo de vida ativo e promovendo a adoção de atividades físicas programadas bem como eventos de lazer ativos (SBP, 2008). Segundo Birch (2002), crianças e adolescentes seguem padróes paternos, que se não forem modificados ou manejados em conjunto, não haverá sucesso na prevenção. Estudos apontam, inclusive, que existem casos de desnutrição e obesidade em uma mesma moradia (GUIMARÁES et al, 2006), o que torna a abordagem familiar ainda mais complexa.

Considerando-se o aumento excessivo do excesso de peso e das doenças crônicas não transmissíveis, é grande o desafio do pediatra de orientar e modificar hábitos alimentares familiares visando à formação de hábitos saudáveis e à promoção de saúde. Praticando alimentação saudável prevenimos alergia alimentar, obesidade, doenças cardiovasculares, dislipidemias, e, estimulando a prática de atividades físicas regulares, diminuímos o sedentarismo, auxiliando a manutenção de um peso adequado.

Segundo Mello (2004), para alcançar uma alimentação saudável, além de fornecer informaçôes corretas sobre alimentação e saúde, é preciso evitar que informações incorretas e contraditórias alcancem indivíduos e, ao mesmo tempo, propiciar a esses indivíduos condiçóes que tornem factível a adoção das orientações que recebem.
Logo, cabe ao pediatra, ainda na atenção básica, orientar a família sobre os passos para uma alimentação saudável, que englobam: o aleitamento materno exclusivo até os seis meses e a introdução de alimentos complementares após este período; a promoção a manutenção da amamentação até os dois anos ou mais; a promoção da alimentação variada e colorida; o consumo diário de frutas, legumes e verduras; a restrição de guloseimas e sal; orientaçôes para higiene no preparo, manuseio, armazenamento e conservação destes; estímulo a alimentação da criança enferma ou em convalescença; acompanhar as curvas de crescimento; dar ênfase aos horários regulares para refeiçóes e lanches; ao consumo de água potável e sucos naturais; orientar a evitar monotonia alimentar; estimular o consumo de gorduras monossaturadas e poliinsaturadas, bem como dos alimentos ricos em ferro, cálcio, vit. A, vit. D e zinco, limitando uso de gorduras trans e saturadas (BRASIL, 2005); além de orientar a higienização da boca mesmo antes do aparecimento dos dentes decíduos (EDMUNDS et. al, 2001).

A adolescência merece especial atenção por tratar-se de uma faixa etária de risco extremamente vulnerável devido ao estilo de vida e alto consumo de energia e gordura cuja quantidade e tipo aumentam os riscos de obesidade, doenças cardiovasculares, resistência insulínica, diabetes tipo 2 e câncer (GIULIANO et. al, 2005; CAMPOS \& ZUANON, 2004). Deve-se afastar ainda a presença de fatores de risco de distúrbios nutricionais como fumo, poucas horas de sono, ingestão de álcool, energéticos e refrigerantes nesta população.

A educação nutricional deve ser realizada em consulta de puericultura e a promoção a alimentação saudável deve ser extensivo às famílias do paciente e a comunidade a qual pertence, pois as famílias preservam, de certa forma, seu modo de cuidar conforme aprendido em seu meio cultural. A questão da obesidade infantil somente será controlada quando houver integração entre o pediatra, a família, a escola (JACOB, 2002; ARAÚJO et. al, 2006) e sociedade. E cabe ao pediatra a tarefa da promoção da saúde infantil, orientando a criaçáo de uma criança saudável para termos adultos saudáveis.

\section{CONCLUSÓES}

O Brasil convive concomitantemente com a desnutrição e com prevalências preocupantes de excesso de peso e obesidade, resultantes da má-alimentação. A puericultura na Atençáo Básica consiste em ferramenta no acompanhamento integral do crescimento e desenvolvimento infantil e deve considerar a criança dentro de uma família e sua cultura. Para 
promover um cuidado em prol da promoção da nutrição infantil, por meio do estímulo a hábitos alimentares saudáveis é de grande relevância o papel do pediatra na vigilância da saúde da criança nos serviços de atenção primária à saúde.

A concretização das açóes de alimentação e nutrição em uma agenda única na atenção primária à saúde é fundamental, assim como a inclusão deste tema na formação superior dos profissionais de saúde, tanto no ensino de graduação, como nos diversos níveis de pós-graduação; a fim de capacitar o profissional para identificar precocemente, realizar intervençóes e encaminhamentos oportunos das crianças com problemas no crescimento e desenvolvimento.

Tratar um paciente obeso com sucesso é sempre um desafio. Logo, a prevenção e programas de intervenção a nível de atenção básica, associados a uma abordagem multidisciplinar são fundamentais para formação de hábitos de vida saudáveis com intuito de reduzir a prevalência da obesidade infantil.

A nutrição infantil é prioridade no horizonte da saúde pública e o pediatra é instrumento fundamental na promoção da saúde e prevenção de doenças. $\mathrm{O}$ aconselhamento de uma alimentação equilibrada e a adoção de um estilo de vida saudável parece ser a melhor maneira para controle da obesidade infantil.

\section{REFERÊNCIAS}

1. ARAÚJO, M.F.M; LEMOS, A.C.S, CHAVES, E.S. Creche comunitária: um cenário para a detecção da obesidade infantil. Ciência, Cuidado e Saúde Maringá, v. 5, n. 1, p. 24-31, 2006.

2. ABRANTES, M.M; LAMOUNIER, J.A; COLOSIMO, E.A. Prevalência de sobrepeso e obesidade em crianças e adolescentes das regióes Sudeste e Nordeste. J Pediatr (Rio J), v.78, p.335-40, 2002.

3. BIRCH, L.L. Childhood Overweight: family environmental factors. In: Chen, C.; Dietz, W.H. Obesity in Childhood and Adolescence. Philadelphia: Lippincott Williams \& Wilkins, p. 161-176, 2002.

4. BRASIL. Ministério da Saúde. Política Nacional de Promoção da Saúde. Série B. Textos básicos de saúde. 1. ed., Brasília, 2006. Disponível em: http://portal. saude.gov.br/portal/arquivos/pdf/pactovolume7.pdf Acesso em 02.06.10.

5. __. Ministério da Saúde. Política nacional de atenção básica. Brasília: Ministério da Saúde; 2006. Disponível em: http://www.saude.gov.br Acesso em 20.06.11.
6. _. Ministério da Saúde. Política nacional de alimentação e nutrição. 2a ed. Brasília: Ministério da Saúde; 2003. Disponível em: http://www.saude.gov.br Acesso em 12.06.11.

7. _ _ Ministério da Saúde. Secretaria de Política de Saúde. Organização pan-americana de saúde. Guia alimentar para crianças menores de dois anos. Brasília, 2005. Disponível em: http://www.saude.gov.br Acesso em 20.06.10.

8. __. Ministério da Saúde. Coordenação-Geral da Política de Alimentação e Nutrição, Departamento de Atenção Básica, Secretaria de Atenção à Saúde. Guia alimentar para a população brasileira: promovendo a alimentação saudável. Brasília, 2006.

9. __ _. Ministério da Saúde. Plano de açóes estratégicas para o enfrentamento das doenças crônicas não transmissíveis (DCNT) no Brasil 2011-2022. Brasília: Ministério da Saúde. Disponível em: http://www.saude. gov.br Acesso em 01.04.12.

10. CAMPOS, J.A.D; ZUANON, A.C.C. Merenda escolar e promoção de saúde. Cienc Odontol Bras, v.7, n.3, p. 67-71, 2004.

11. CASTRO, F.A.F; PEREIRA, C.A.S; PRIORE, S.E; RIBEIRO, S.M.R; BITTENCOURT, M.C.B; QUEIROZ, V.M.V. Educação Nutricional: a importância da prática dietética. Nutrição em Pauta, v.10, n.52, p.9-15, 2002.

12. CORSO, A. C. T. et. al. Sobrepeso em crianças menores de 6 anos de idade em Florianópolis, SC. Revista de Nutrição, v. 16, n. 1, p. 21-28, 2003.

13. COUTINHO, J.G; GENTIL, P.C; TORAL, N. A desnutrição e obesidade no Brasil: o enfrentamento com base na agenda única da nutrição. Cad. Saúde Pública, 24 Sup 2: S332-S340, 2008.

14. DIETZ, W.H. The obesity epidemic in young children. BMJ, v. 322, n.7282, p.313-314, 2001.

15. EBBELING, C.B; PAWLAK, D.B; LUDWIG, D.S. Childhood obesity: publichealth crisis, common sense cure. Lancet, v.360, n.9331, p. 473- 482, 2002.

16. EDMUNDS, L; WATERS, E; ELLIOTT, E.J. Evidence based pediatrics: evidence based management of childhood obesity. BMJ, v. 323, n.7318, p.916-919, 2001. 
17. ESCRIVÃO, M.A; TADDEI, J.A. Obesidade na infância e adolescência. In: Nóbrega, F.J. Distúrbios da Nutrição na Infância e na Adolescência. Rio de janeiro: Revinter, 2007.

18. FIATES, G.M.R.; AMBONI, R.D.M.C.; TEIXEIRA, E. Comportamento consumidor, hábitos alimentares e consumo de televisão por escolares de Florianópolis. Revista de Nutrição, v.21. n.1, p.105-114, 2008.

19. FIGUEIRAS, A.C.M; PUCCINI, R.F; SILVA, E.M.K; PEDROMÔNICO, M.R.M. Avaliação das práticas e conhecimentos de profissionais da atenção primária à saúde sobre vigilância do desenvolvimento infantil. Cad Saúde Pública, v.19, n6, p.1691-1699, 2003.

20. FILGUEIRAS, M.C; LIMA, N.V.R; SOUZA, S.S; MOREIRA, A.K.F. Prevalência de obesidade em crianças de escolas públicas. Revista Ciência \& Saúde, Porto Alegre, v. 5, n. 1, p. 41-47, 2012.

21. FAGLIOLI, D. NASSER, L. A. Educação nutricional na infância e na adolescência: planejamento, intervenção, avaliação e dinâmicas. São Paulo, SP: RCN, 2008.

22. GIULIANO, I.C.B; CARAMELLI, B; PELLANDA. L; et. al. I Diretriz de Prevenção da Aterosclerose na Infância e Adolescência. Arq. Bras Cardiol, v. 85, p.34-36, 2005.

23. JACOB, A. School programs. In: Chen, C.; Dietz, W.H. Obesity in Childhood and Adolescence. Philadelphia: Lippincott Williams \& Wilkins, p. 257-72, 2002.

24. GUIMARÃES LV, BARROS MBA, MARTINS MSAS, DUARTE EC. Fatores associados ao sobrepeso em escolares. Rev Nutrição, v.19, n.1, p. 5-17, 2006.

25. JAIME, P.; SILVA, A.C.F.; LIMA, A. M. C.; BORTOLINI, G.A. Ações de alimentação e nutrição na atenção básica: a experiência de organização no Governo Brasileiro. Rev. Nutr. [online], v. 24, n.6, p. 809-824, 2011.

26. LOPES, P. C. S; PRADO, S. R. L.A.; COLOMBO, P. Fatores de risco associados à obesidade e sobrepeso em crianças em idade escolar. Rev. bras. enferm. (on line), v.63, n.1, p. 73-78, 2010.

27. MARCONDES, E. Diretrizes para o ensino da pediatria. J Pediatr (Rio J), v.69, p. 349-52, 1993.
28. MELLO, E.D; LUFT, V.C; MEYER, F. Obesidade infantil: como podemos ser eficazes? J Pediatr (Rio J), v.80, n.3, p173-182, 2004.

29. MIRANDA, A.A.N; NAVARRO, F. A prevenção e o tratamento da obesidade durante a infância: uma opção eficaz para reduzir a prevalência desta patologia. Revista Brasileira de Obesidade, Nutrição e Emagrecimento, São Paulo v.2, n.10, p.313-323, 2008.

30. MONTEIRO, C.A; CONDE, W.L. Tendência secular da desnutrição e da obesidade na infância na cidade de São Paulo (1974-1996). Revista Saúde Pública, v.34, p. 52-61, 2007.

31. OLIVEIRA, C.L.; FISBERG, M. Obesidade na infância e adolescência: uma verdadeira epidemia. Arquivos Brasileiros de Endocrinologia e Metabologia, v.47, n. 2, p.107-108, 2003.

32. OMS. ORGANIZAÇÃO MUNDIAL DA SAÚDE. Declaração de Alma-Ata. Conferência Internacional sobre Cuidados Primários de Saúde - Alma-Ata. URSS, 6-12 de setembro de 1978.

33. PEREIRA, L. Obesidade na adolescência: a importância de bons hábitos alimentares. Adolescência \& Saúde, v.3, n. 1, 2006.

34. QUEIROZ, M. V.; JORGE, M. S. Health education strategies and the quality of care and teaching in pediatrics: interaction, connection and trust in professional discourse. Interface - Comunic., Saúde, Educ., v.10, n.19, p.117-130, 2006.

35. REICHERT, A.P.S; ALMEIDA, A.B; SOUZA, L.C; SILVA, M.E.A; COLLET, N. Vigilância do crescimento infantil: conhecimento e práticas de enfermeiros da atenção primária à saúde. Rev. Rene, v.13, n.1, p.114-126, 2012.

36. RODRIGUES, E.M.; BOOG, M.C.F. Problematização como estratégia de educação nutricional com adolescentes obesos. Caderno de Saúde Pública, v.22, n.5, p.923- 931, 2006.

37. SAHOTA, P.; RUDOLF, M.C.J; DIXEY, R; HILL, A.J; BARTH, J.H; CADE, J. Evaluation of implementation and effect of primary school based intervention to reduce risk factors for obesity. BMJ, v. 323, p.1-4, 2001. 
38. SBP. Sociedade Brasileira de Pediatria. Departamento de Nutrologia. Manual de orientação de obesidade na Infância e Adolescência. SBP, São Paulo, 2008. Disponível em: <http://www.sbp.com.br >Acesso em 20.06.10.

39. Sociedade Brasileira de Pediatria, São Paulo. Departamento de Nutrologia. Manual de orientação: alimentação do lactente, do pré-escolar, escolar, adolescente e na escola. SBP, 2008. Disponível em: < http:// www.sbp.com.br >Acesso em 20.06.10.

40. SILVA, C.P.G; BITTAR, C.M.L. Fatores ambientais e psicológicos que influenciam na obesidade infantil. Revista Saúde e Pesquisa, v. 5, n. 1, p. 197-207, 2012.

41. TRICHES, R.M.; GIUGLIANI, E.R.J. Obesidade, práticas alimentares e conhecimentos de nutrição em escolares. Revista de Saúde Pública, v.39, n.4, p.541-547, 2005.

42. UAUY, R; MONTEIRO, C. The challenge of improving food and nutrition in Latin America. Food Nutr Bull, v. 25, p.175-82, 2004.

43. WANG, Y; MONTEIRO, C; POPKIN, B.M. Trends of obesity and underweight in older children and adolescents in the United States, Brazil, China, and Russia. Am J Clin Nutr., v. 75, n.6, p. 971-917, 2002.

44. YANOVSKI, S.Z; YANOVSKI, J.A. Obesity. N Engl J Med, v.346, n.8, p. 591-602, 2002. 